\title{
Effects of dietary folate intake on migraine disability and frequency.
}

Saras Menon, $\mathrm{PhD}^{1}$, Rodney Arthur Lea, $\mathrm{PhD}^{1,2}$, Sarah Ingle, MSC ${ }^{2}$, Michelle Hanna, $\mathrm{BSC}^{1,2}$, Shirley Wee, $\mathrm{Phd}^{2}$, Michelle Palmer, $\mathrm{PhD}^{2}$, and Lyn Robyn Griffiths, $\mathrm{PhD}^{1}$

${ }^{1}$ Genomics Research Centre, Institute for Health and Biomedical Innovation, Queensland University of Technology, Corner of Musk Avenue and Blamey Street, Kelvin Grove, Queensland, Australia, 4059.

${ }^{2}$ Griffith Health Institute, Griffith University, Gold Coast, Parklands Drive, Southport, Queensland, Australia, 4222

\section{Conflict of Interest:}

The authors declare that they have no conflicting interest.

\section{Financial support:}

This study was supported by funding from the Nutrica Research Foundation project grant and the Department of Innovation, Industry, Science and Research- Funding agreement:

International Science Linkages grant

\section{Communicating Author:}

Professor Lyn Griffiths,

Executive Director, Institute of Health and Biomedical Innovation

Queensland University of Technology

Musk Ave, Kelvin Grove, Queensland, 4059, Australia.

Telephone: +61731386102

Fax: +61731386039

Email: 1yn.griffiths@qut.edu.au 


\section{Abstract}

Migraine is a highly disabling disease affecting a significant proportion of the Australian population. The Methylenetetrahydrofolate Reductase (MTHFR) C677T variant has been associated with increased levels of homocysteine and risk of migraine with aura (MA). Folic acid, Vitamin $\mathrm{B}_{6}$ and $\mathrm{B}_{12}$ supplementation has been previously shown to reduce increased levels of homocysteine and decrease migraine symptoms. However the influence of dietary folate intake on migraine has been unclear. The aim of the current study was to analyse the association of dietary folate intake in the form of dietary folate equivalent (DFE), folic acid (FA) and total food folate (TFF) on migraine frequency, severity and disability. A cohort of 141 adult females of Caucasian descent with MA was genotyped for the MTHFRC677T variant using restriction enzyme digestion. Dietary folate information was collected from all participants and analysed using the "FoodWorks" 2009 package. Folate consumption was compared to migraine frequency, severity and disability using linear regression. A significant inverse relation was observed between DFE $\left[\mathrm{R}^{2}=0.201, P=0.045\right.$, CI $\left.(-0.004,-0.001)\right]$ and FA $\left[\mathrm{R}^{2}=0.255, P=0.036, \mathrm{CI}(-0.009,-0.002)\right]$ consumption and migraine frequency. It was also observed that in individuals with the CC genotype for the MTHFR C677T variant, migraine frequency was significantly linked to FA consumption $\left[\mathrm{R}^{2}=0.077, P=0.029\right.$, CI ($0.009,0.005)]$.

\section{Key Words:}

MA, MTHFRC677T, Folic acid, Dietary folate equivalent, Homocysteine 


\section{Introduction}

Migraine is an extremely debilitating and highly prevalent neurovascular disorder presenting multiple symptoms(de Vries, 2009 \#686). Migraine affects a significant proportion of the Australian population, thus placing a large economic burden on the economy(Stovner, 2007 \#824). The International Headache Society (IHS) has subdivided migraine into two broad categories of migraine with aura (MA) and migraine without aura (MO)(Society, 2004 \#562). MA has been linked to the Methylenetetrahydrofolate reductase gene mutation C677T(Kowa, 2000 \#128; Lea, 2004 \#548). Individuals with the MTHFR C677T variant have shown diminished capacity to remethylate homocysteine to methionine, consequently inducing the increase in circulating plasma homocysteine levels (Di Rosa, 2007 \#448; Kara, 2003 \#121; Kowa, 2000 \#128; Lea, 2004 \#548). Elevated homocysteine levels have been shown to be an independent risk factor for cardiovascular disease as well as being associated with endothelial dysfunction (Aguilar, 2004 \#825; Klerk, 2002 \#826). While alteration in vascular function and cerebral blood flow has been identified in MA sufferers, the exact role vascular dysfunction plays in migraine pathogenesis is yet to be unravelled (Moschiano, $2008 \# 827$; Johnson, 1978 \#771).

The B group vitamins are crucial co-enzymes in the maintenance of homocysteine homeostasis via the Methylation Cycle and deficiencies in these vitamins have been implicated in hyperhomocysteinemia(Silaste, 2001 \#238). Folic acid, $\mathrm{B}_{6}$ and $\mathrm{B}_{12}$ supplementation have been previously shown to reduce migraine disability, frequency and severity in MA sufferers (Di Rosa, 2007 \#448; Lea, 2009 \#137; Menon, 2012 \#812). However the effects of dietary B vitamin consumption on migraine severity, frequency and disability are yet to be determined. The aim of the current study was to analyse the 
consumption of dietary folate among a cohort of adult females with MA and to determine if there is an association between the levels of folate consumption, MTHFR genotype, migraine frequency, severity and disability prevalence.

\section{Methods}

\section{Study Design and participant group}

This study involved the secondary analysis of data collected from a randomised, double blind, placebo controlled clinical trial on the effects of vitamin B supplementation and MTHFR and Methionine Synthase Reductase (MTRR) genotype on migraine disability, severity and frequency (Menon, 2012 \#812). The intervention period for this trial was 6 months with serum folate and plasma homocysteine levels recorded at baseline and at the end of the trial. MTHFR genotype was determined and migraine disability and frequency was also recorded. Participants of the clinical trial were asked to complete diet diaries which were used to analyse the composition of dietary folate intake [Total Food Folate (TFF), Folic Acid (FA) and Dietary Folate Equivalents (DFE)]. Pretrial data (Baseline measurement) from the participants was used for the dietary analysis. The baseline folate intake of the participants was compared to the baseline measurements of plasma homocysteine, serum folate, MTHFR C677T variant, migraine severity, frequency and disability. The study was originally approved by the Griffith University Ethics Committee for experimentation on human subjects.

\section{Patient group}

The study recruited 245 Caucasian females with MA of European ancestry from Australia between the ages of 18 and 65 . A detailed inclusion criterion for participant recruitment for the migraine trial is described in the study by Menon.S et al 2012(Menon, 2012 \#812). 
Briefly participants were included if they had suffered from migraine for more than 5 years and had a current diagnosis of MA ( $>90 \%$ of their migraine were associated with aura), and a 1 year history of severe, long-lasting attacks (at least 4 attacks) and had a family history of migraine. Participants were excluded if they were pregnant or suffered from a clinically recognised cardiovascular or neuropsychiatric condition. All participants were instructed to cease all vitamin supplementation 2 weeks prior to commencement of the trial (Menon, 2012 \#812).

\section{Baseline Assessment and genotyping}

Total fasting plasma homocysteine, and serum folate concentrations were taken at baseline and at the conclusion of the six month trial intervention. The plasma homocysteine $(\mu \mathrm{mol} / \mathrm{l})$, serum folate $(\mathrm{nmol} / \mathrm{l})$, Serum $\mathrm{B}_{6}(\mathrm{nmol} / \mathrm{L})$ and serum $\mathrm{B}_{12}(\mathrm{pmol} / \mathrm{L})$ were measured in an accredited laboratory. Genotype assessment was performed by extracting DNA from the participants' blood and analysed using standard techniques described in Lea et al 2004(Lea, 2004 \#139). Participants were assessed for migraine disability using the Migraine Disability Assessment Score (MIDAS) Instrument, which provides a measure of productive days lost to migraine headache in the previous 3 months. Questions 6 and 7 of the MIDAS instrument were on migraine frequency and head pain severity respectively. Participants were asked to complete a MIDAS prior to starting the trial, 3 months after starting the trial and the end of the 6 month intervention of the trial.

\section{Dietary Assessment Tool}

The dietary assessment tool used in this trial was designed to focus on foods that were rich sources of folate, $>200 \mathrm{ug} / 100 \mathrm{~g}$ according to the Nutrient Tables for use in Australia (NUTTAB) 2006 Composition Table and was created to document the subject's daily food 
consumption, with an emphasis on folate consumption. A day consisted of a breakfast, lunch, dinner and snack section. Participants were instructed to indicate what they ate at each corresponding meal time and to write down "other food" items that they had consumed that was not on the list of the foods given in the diet diary provided. Subjects were advised that one serve of food was equal to $100 \mathrm{~g}$ and were instructed to indicate if fruits and vegetable consumed were cooked (Fried, boiled, baked) or raw. Participants were advised to complete one diary before commencing the trial and one diary for every fortnight of the trial duration.

\section{Diet Diary Analysis}

Analysis of estimated dietary folate intake was performed using the nutrient analysis software FoodWorks Professional (Version 6, 2009, Xyris Software, Brisbane, Australia). The TFF, FA and DFE content of each diet diary completed by each participant was analysed. In instances where participants did not specify cooking techniques an unspecified option (Not Specified-NFS) was always selected. If serving sizes were not specified, a 'Standard' serving size was selected as specified by FoodWorks. In instances where FoodWorks did not have a specific food item in its data base, the closest possible option was selected. All assumptions were made to be as consistent as possible. Diet diary data was excluded if folate intake was not reported within two weeks of relevant biomarker measurements.

\section{Assessment of Folate Intake}

Total folate intakes were computed using data from diet diary. The bioavailability of food folate is lower than that of folic acid added to fortified foods and dietary supplements. The DFE conversion of TFF was therefore used to account for differential bioavailability. In Australia, the National Health and Medical Research Council (NHMRC, 2006) calculate DFE as: 
$\mathrm{DFE}=($ Food Folate $\mu \mathrm{g})+($ Folic acid $\mu \mathrm{g} \times 1.67)$

DFE, TFF and FA were used in the analysis of folate intake in this study.

\section{Statistical Analysis}

All analyses were performed using the Statistical Package for Social Sciences (SPSS version 21.0). Average dietary folate (TFF, FA and DFE) serum folate and plasma homocysteine concentration means were determined by descriptive analysis. The relationship between migraine clinical variables (migraine frequency, MIDAS and migraine pain severity), biochemical variables (Serum folate, serum $\mathrm{B}_{6}, \mathrm{~B}_{12}$ and plasma folate) and dietary folate consumption (DFE, FF and TFF) was analysed using Pearson's correlation test. Linear regression analysis was performed using the "forward conditional" method and the "Enter" method, to determine the independent predictors of migraine frequency, severity and disability, allowing the introduction of a new variable if the $P$ value of the new model was less than 0.05 , and excluding those yielding a $P$ value higher than 0.10 in each step. Significance thresholds for all statistical tests were set at $P<0.05$ level.

\section{Results}

Diet diaries were successfully collected from 141 participants who were then genotyped for the MTHFRC677T polymorphism (Table 1). Biomarker correlations were only performed on diet diaries that were completed within two weeks of blood tests. The dietary folate intake of the participants calculated using foodworks was $551.78 \pm 19.7$ DFE and well above the Required Daily Intake (RDI) of $400 \mu \mathrm{g}$ DFE. The ratio (\%) of individuals who consumed more than RDI of DFE $400 \mu \mathrm{g}$ were $70.2 \%(\mathrm{n}=99)$. The DFE intake ranges from $161 \mu \mathrm{g}$ to $1286 \mu \mathrm{g}$. The mean homocysteine level for the population tested in this study was 11.65 $\mu \mathrm{mol} / 1$. A positive Pearson's correlation was found between DFE consumption and 
serum folate $(\mathrm{r}=0.209, P=0.019)$. An inverse positive correlation was also observed between DFE consumption and migraine frequency $(\mathrm{r}=-0.200, P=0.024)$. MIDAS was positively correlated to migraine pain severity $(\mathrm{r}=0.175, P=0.039)$. An inverse significant relation was also observed between serum folate levels and plasma homocysteine levels $(\mathrm{r}=\mathrm{-}$ $0.276, \mathrm{P}=0.002)$.

Regression analysis was used to test if folate consumption and the MTHFR genotype significantly predicted serum folate levels. The results of the regression indicated that DFE consumption predicted $4.4 \%$ of the variance $\left[\mathrm{R}^{2}=0.044, P=0.019,95 \% \mathrm{CI}(0.002,0.20)\right]$, FA consumption predicted $3.7 \%$ of the variance $\left[\mathrm{R}^{2}=0.037, P=0.059,95 \% \mathrm{CI}(-0.01,0.020)\right.$ and TFF consumption predicted $2.6 \%$ of the variance in serum folate levels $\left(\mathrm{R}^{2}=0.026, P=0.073\right.$, 95\% CI (-0.001, 0.023)]. Folate consumption models were also run including MTHFR genotype as a possible confounding factor in a multivariate analysis; however the MTHFR genotype was not a significant contributor for serum folate levels.

Univariate analysis was performed on variables: $\mathrm{B}_{6}, \mathrm{~B}_{12}$, serum folate and the MTHFR C677T genotype to determine significant predictors of plasma homocysteine levels. Serum $\mathrm{B}_{12}\left[\mathrm{R}^{2}=0.053\right.$, CI $\left.(-0.011,-0.002) P=0.007\right]$ and serum folate $\left[\mathrm{R}^{2}=0.165\right.$, CI $(-0.222$, 0.048) $P=0.003$ ] levels were significant factors in determining plasma homocysteine levels. However when multivariate analysis was performed including all variables in a step wise regression, only serum folate was found to be a significant factor of plasma homocysteine levels $\left[\mathrm{R}^{2}=0.173, \mathrm{~B}=-0.122, P=0.063,95 \% \mathrm{CI}(-0.219,-0.024)\right]$. Folate consumption (DFE, FA and TFF) were not significantly related to plasma homocysteine levels $(\mathrm{P}>0.05)$ 
When folate consumption and migraine outcomes were tested in univariate regression analysis, it was observed that DFE consumption $\left[\mathrm{R}^{2}=0.040, P=0.024, \mathrm{CI}(-0.002,0.002)\right]$ and in particular FA consumption $\left[\mathrm{R}^{2}=0.080, P=0.004\right.$, CI $\left.(-48.818,2.989)\right]$ was significantly related to migraine frequency (Table 2).In multiple regression analysis, DFE and FA consumption adjusted for $\mathrm{B}_{6}, \mathrm{~B}_{12}$, plasma homocysteine levels and the MTHFR genotype, were significantly related to migraine frequency (Table 3). Serum folate levels adjusted for serum $\mathrm{B}_{6}$ and $\mathrm{B}_{12}$, plasma homocysteine levels and the MTHFR genotype were not significantly related to migraine frequency $\left[\mathrm{R}^{2}=0.025, P=0.956\right.$, CI $\left.(-0.04,0.031)\right]$.

When data was stratified by the MTHFR genotype, it was observed that $\mathrm{T}$ allele carriers had consumed the most amounts of DFE (561.3ug) and TFF(486.83ug) but the least amount of FA(139.8ug) compared to the CC genotype carriers (DFE: 541.3ug, TFF: 455.13ug, FA: 161.62ug). It was also observed that the $\mathrm{T}$ allele carriers had higher plasma homocysteine levels $(12.0 \mu \mathrm{mol} / \mathrm{L})$ and lower serum $\mathrm{B}_{12}(309.03 \mathrm{pmol} / \mathrm{L})$ and serum folate levels $(29.1 \mathrm{nmol} / \mathrm{L})$ compared to the $\mathrm{CC}$ genotype carriers (Homocysteine: $11.4 \mu \mathrm{mol} / \mathrm{L}, \mathrm{B}_{12}$ : 314.36pmol/L, Serum Folate: 30.3.nmol/L). The percentage of $\mathrm{T}$ allele carriers experiencing an average of 3 to 6 migraines in 3 months was $32.4 \%$ compared to $24.5 \%$ in the CC genotype carriers. Further regression analysis observed that in individuals with the CC genotype, migraine frequency was significantly inversely related to FA consumption $[R 2=$ $0.077, P=0.029$, CI $(-0.009,0.005)]$ (Table 4).

Univariate analysis and multivariate analysis adjusted for $\mathrm{B}_{6}, \mathrm{~B}_{12}$, plasma homocysteine levels and the MTHFR C677T genotype did not identify a significant relationship between folate consumption (DFE, FA, TFF) or serum folate levels and migraine associated disabilities such MIDAS and migraine pain severity scores $(\mathrm{P}>0.05)$. 


\section{Discussion}

A possible interplay between genetic and environmental factors has been the basis of migraine pathophysiology hypothesised to date(Mulder, 2003 \#184). Recent studies have reported a higher incidence of the co-occurrence of ischemic cardiocerebrovascular disease (CVD) and migraine(Elliott, 2008 \#829; Kruit, 2004 \#132; Kurth, 2005 \#135; Kurth, 2006 \#830; Kurth, 2006 \#831). This incidence seems to be increased in MA sufferers(MacClellan, 2007 \#157). Elevated plasma Hcy levels have been reported to increase the risk of atherosclerotic vasculopathy and act as an independent risk factor for CVD(Boushey, 1995 \#828). An increase in total Hcy concentration has also been recently reported in migraineurs specifically in those with MA(Isobe \#822). It is plausible that homocysteine levels may be involved in both MA and CVD pathophysiology. Reasons for hyperhomocysteinemia may include mutations in homocysteine metabolising genes such as MTHFR, MTRR and Cystathionin Beta Synthase (CBS) or possible nutritional deficiencies in cofactors involved in homocysteine metabolism (Silaste, 2001 \#238).

The current study investigated the association between dietary folate consumption, MTHFR genotype and migraine associated disability in a cohort of female MA sufferers. This may be the only study of its kind to analyse food folate consumption by examining folate consumption in three different components: Total Food Folate (TFF), the fortified folic acid (FA) content of foods and the Dietary folate equivalent (DFE) in food consumed in migraineurs. The results of this study observed a significant link between increasing serum folate levels and increasing DFE consumption and this significance most probably contributed to the total consumption of FA more than TFF. However the results also show that DFE consumption and FA consumption only account for $4.4 \%$ and $3.7 \%$ respectively, of serum folate levels. Serum folate levels were significantly correlated to decreasing plasma 
homocysteine levels as expected in the current study, however there was no significant link observed between serum folate levels and migraine frequency, MIDAS or pain severity. Folate mainly taken up by developing erythrocytes reflects time integrated intake and is therefore considered to be a measure of long term folate status, while serum folate predominantly reflects transitory changes in folate concentration(, 1994 \#840). Red blood cell folate may have provided a more accurate measurement of folate levels in the participants of this trial compared to serum folate measurement. Other influencing factors of serum folate levels are the differing acute post-absorptive plasma kinetics of folate and the challenges in accurately determining dietary folate bioavailability (Ohrvik, \#839).

In the migraine population tested in the current study, a significant correlation between folate consumption and plasma homocysteine levels was not observed. This finding is contrary to many previous studies that have observed a significant inverse relationship between folate consumption and homocysteine levels in plasma(, 1998 \#843; Wald, 2001 \#844; Ward, 1997 \#842). The "FoodWorks" software utilised in this trial did not have several food items that were popular amongst the participants of this trial. The closest food match was selected, for example, "Raw spinach" was selected in place of rocket. This may have affected folate consumption measurement as food substitutes used may not have been the most accurate comparisons. Secondly the lack of confounding factors analysed in the current trial may have also influenced the lack of association observed between plasma homocysteine levels and dietary folate intake. The study did not collect demographic information such as participant smoking habits, blood pressure or blood cholesterol levels. Dyslipidemia, hypertension and smoking have been shown to effect serum folate and plasma homocysteine levels (El Oudi, \#845). 
A major finding of this study is the significant inverse relation observed between DFE and more specifically FA consumption and migraine frequency. It was also observed that in individuals with the CC genotype for the MTHFR C677T variant, increasing levels of FA consumption significantly related to decreasing migraine frequency. $\mathrm{T}$ allele carriers who had on average consumed less FA than CC genotype carriers showed no correlation between FA consumption and migraine frequency. Although the current study did not find a significant correlation between the MTHFR C677T genotype and migraine frequency or homocysteine levels, it was observed that the $\mathrm{T}$ allele carriers, albeit by a small margin, had higher migraine frequency and plasma homocysteine levels compared to individuals with the CC genotype for the MTHFR C677T variant. Alternatively, mean serum $\mathrm{B}_{12}$ and folate levels were also observed to be lower in $\mathrm{T}$ allele carriers compared to the $\mathrm{CC}$ genotype carriers.

Naturally occurring folate found in food is present in the reduced, polyglutamated form with methyl or formyl as the one carbon substitution (Perry, 1970 \#846; Scott, 1976 \#847). Folic acid found in fortified food and supplements, is a synthetic, fully oxidised monoglutamate form of folate and is reduced to tetrahydrofolate prior to its participation in a metabolic reaction (Futterman, 1957 \#850). Relative to naturally found folate, folic acid has a higher bioavailability, which is defined as the proportion of ingested folate that is absorbed and can be used for metabolic processes (Caudill, \#849). Although there is a broad consensus that folic acid is more bioavailable than naturally found food folate, there are some intervention trials with food that have reported similar improvements in folate status compared with equimolar or small doses of folic acid from fortified foods or supplements(Fenech, 2005 \#854; Vahteristo, 2002 \#851; Winkels, 2007 \#852). 
Previous clinical trials by Lea et al 1999 and Menon at al 2011 have provided evidence that homocysteine lowering by folic acid supplementation can reduce migraine disability and that this effect may be modified by polymorphisms in genes coding for key enzymes that affect folate metabolism, such as the MTHFR genotype (Lea, 2009 \#137; Menon, 2012 \#812). T allele carriers of the MTHFR C677T genotype with a $35 \%$ to $70 \%$ reduction in their enzymatic rate are genetically slower in homocysteine metabolism (Frosst, 1995 \#81). The findings of the current study, as well as those from previous migraine trials, suggest that individuals who carry the T allele for the MTHFR C677T variant, may require an increase in folate consumption, specifically the more bioavailable FA(Ashfield-Watt, 2002 \#832; Hickling, $2005 \# 833$ ), to experience reduced migraine frequency and possibly other associated symptoms as well (Lea, 2009 \#137; Menon, 2012 \#812).

Certain limitations of this study include the small participant sample size which may not offer a good representation of the female Caucasian population suffering from MA and their dietary folate levels. It should also be noted that the dietary information from the migraineurs of the current study was also obtained prior to the implementation of the mandatory folate fortification in Australia. Mandatory folate fortification was implemented in Australia in September 2009. Brown et al reported a significant increase in serum folate levels between prefortification folate serum concentrations and serum folate concentrations taken approximately 6 months post fortification (Brown \#823). A recent study by Dugbaza et al 2012 that investigated the levels of total dietary folic acid intake following the mandatory folic acid fortification reported an increase of 2.5 times in folic acid consumption in the Australian adult population(Dugbaza \#834). Among females with low intake of folate (represented by the tenth percentile of intake), mandatory fortification has increased their estimated intake from less than $5 \%$ to $75 \%$ of the recommended amount (Dugbaza \#834) 
The premise of mandatory folate fortification policy was to reduce the incidence of Neural Tube Defects: however secondary health benefits may result, such as the potential role of decreasing homocysteine concentrations in reducing cardiovascular disease as well as migraine frequency and disability. A long term follow up population study is necessary to investigate if mandatory folate fortification results in a decrease in migraine incidence in Australia and if it successfully translates to a decrease in migraine associated disability (work place absenteeism due to migraine) and positively impacts on the financial burden of migraine in the country's economy.

\section{Conclusion}

An increase in dietary folate, especially the more bioavailable FA consumption levels may effectively reduce migraine frequency in female migraineurs. The $\mathrm{T}$ allele carriers of the MTHFR C677T variant may need higher levels of FA compared to CC genotype carriers to experience a significant reduction in their migraine frequency.

\section{Acknowledgements}

We would like to acknowledge all participants who consented and volunteered to be involved in this study. L.Griffiths was involved in the conception and design of the study. M.Hanna S.Menon and S. Wee were involved in the generation and collection of data. M.Palmer, S.Hingle and S.Menon were involved in the assembly of data. R.lea, S.Hingle and S.Menon were involved in the analysis and interpretation of the data. L.Haupt, R.lea and S.Menon were involved in the drafting and revision of the manscript. L.Griffiths and R.Lea were involved in approving the final version of the manuscript. This study was supported by funding from the Nutrica Research Foundation project grant and the Department of 
Innovation, Industry, Science and Research- Funding agreement: International Science Linkages grant. R.L was supported partially by a Corbett Research Fellowship. Experiments comply with current laws in Australia.

\section{References}


Table 1: Allele and genotype frequencies for the MTHFRC677T in females with MA in the current study.

\begin{tabular}{|c|c|c|c|c|c|}
\hline \multirow[b]{2}{*}{ MTHFR C677T } & \multicolumn{2}{|c|}{ Allele Frequency } & \multicolumn{3}{|c|}{ Genotype Frequency } \\
\hline & $\mathrm{C}$ & $T$ & CC & CT & TT \\
\hline & 181 & 101 & 63 & 55 & 23 \\
\hline
\end{tabular}

Methylenetetrahydrofolate reductase gene variant (MTHFR C677T) genotype and allele frequencies in migraine with aura (MA) participants of the current study. 
Table 2: Folate consumption predictors of migraine frequency in univariate regression analysis.

\begin{tabular}{llllll}
\hline Model & $\mathrm{R}^{2}$ & $\mathrm{~B}$ & $P$ & $\begin{array}{c}95 \% \mathrm{CI} \\
\text { Lower }\end{array}$ & Higher \\
& & & & & \\
\hline DFE & 0.040 & -0.001 & $0.024^{*}$ & -0.002 & 0.002 \\
\hline FA & 0.080 & -0.004 & $0.004^{*}$ & -0.006 & -0.001 \\
\hline TFF & 0.024 & -22.915 & 0.080 & -48.818 & 2.989 \\
\hline
\end{tabular}

Univariate regression analysis of folate consumption and migraine frequency showed significant links between Dietary folate equivalent (DFE) and Folic acid (FA) consumption and migraine frequency. $95 \% \mathrm{CI}=95 \%$ confidence interval. ${ }^{*}$ Significance was taken at $\mathrm{P} \leq$ 0.05 . 
Table 3: Linear regression analysis: Influence of folate consumption on migraine frequency, adjusted for $\mathrm{B}_{6}, \mathrm{~B}_{12}$, plasma homocysteine levels and MTHFR genotype.

\begin{tabular}{lllllll}
\hline Model & Model R & Model $P$ & $\mathrm{~B}$ & $P$ & \multicolumn{2}{l}{$95 \% \mathrm{CI}$} \\
& & & & & Lower & Higher \\
\hline $\mathrm{DFE}^{\epsilon}$ & 0.201 & $0.045^{*}$ & -0.002 & $0.001^{* *}$ & -0.004 & -0.001 \\
\hline $\mathrm{FA}^{€}$ & 0.255 & $0.036^{*}$ & -0.005 & $0.002^{* *}$ & -0.009 & -0.002 \\
\hline $\mathrm{TFF}^{\epsilon}$ & 0.152 & 0.141 & -0.003 & $0.007^{* *}$ & -0.005 & -0.001 \\
\hline
\end{tabular}

Linear regression analysis of folate consumption adjusted for $\mathrm{B}_{6}, \mathrm{~B}_{12}$, plasma homocysteine levels and the Methylenetetrahydrofolate reductase gene variant (MTHFR C677), showed significant links between Dietary folate equivalent (DFE) and Folic acid (FA) consumption and migraine frequency. $95 \% \mathrm{CI}=95 \%$ confidence interval. *Significance was taken at $\mathrm{P} \leq$ 0.05. ${ }^{€}$ Adjusted for $\mathrm{B}_{6}, \mathrm{~B}_{12}$, plasma homocysteine levels and MTHFR genotype 
Table 4: Linear regression analysis exploring the influence of folate consumption on migraine frequency, when stratified by the MTHFR C677T genotype.

\begin{tabular}{llllllll}
\hline Model & & $\mathrm{R}^{2}$ & $P$ & $\mathrm{~B}$ & \multicolumn{2}{l}{$95 \% \mathrm{CI}$} \\
& & & & & Lower & Higher \\
\hline CC & DFE & 0.058 & 0.069 & -0.001 & -0.002 & 0.0009 \\
& FA & 0.106 & $0.029^{*}$ & -0.004 & -0.007 & 0.0004 \\
& TFF & 0.016 & 0.156 & -0.001 & -0.003 & 0.0005 \\
\hline CT/TT & DFE & 0.028 & 0.176 & -0.001 & -0.002 & 0.0004 \\
& FA & 0.061 & 0.071 & -0.004 & -0.008 & 0.0003 \\
& TFF & 0.016 & 0.301 & -0.001 & -0.003 & 0.001 \\
\hline
\end{tabular}

Linear regression analysis of folate consumption and migraine frequency, stratified by the Methylenetetrahydrofolate reductase gene variant C677T (MTHFR C677T) showed that in individuals with the $\mathrm{CC}$ genotype, migraine frequency is inversely related to $\mathrm{FA}$ consumption. $95 \% \mathrm{CI}=95 \%$ confidence interval. * Significance was taken at $\mathrm{P} \leq 0.05$. 